\title{
FREE POISSON HOPF ALGEBRAS GENERATED BY COALGEBRAS
}

\author{
A. L. AGORE
}

\begin{abstract}
We construct the analogue of Takeuchi's free Hopf algebra in the setting of Poisson Hopf algebras. More precisely, we prove that there exists a free Poisson Hopf algebra on any coalgebra or, equivalently that the forgetful functor from the category of Poisson Hopf algebras to the category of coalgebras has a left adjoint. In particular, we also prove that the category of Poisson Hopf algebras is a reflective subcategory of the category of Poisson bialgebras. Along the way, we describe coproducts and coequalizers in the category of Poisson Hopf algebras, therefore showing that the latter category is cocomplete.
\end{abstract}

\section{INTRODUCTION}

A Poisson Hopf algebra is both a Poisson algebra and a Hopf algebra such that the comultiplication and the counit are morphisms of Poisson algebras. Such objects are situated at the border between Poisson geometry [9] and quantum groups [6]. Poisson structures are known for making striking and unexpected appearances in a variety of different fields of mathematics or mathematical physics such as differential geometry, both classical and quantum mechanics, Lie groups and representation theory, algebraic geometry, etc. Furthermore, as it turns out, many important objects carry not only a Poisson structure but also a natural Poisson Hopf algebra structure; we only mention here the algebra of smooth functions on a Poisson group or the polynomial algebra $\mathbb{C}\left[\mathfrak{g}^{*}\right]$ of a finite dimensional complex simple Lie algebra $\mathfrak{g}$, see e.g. $[6,7,9,22]$ for more details and further examples. On the other hand, there are also examples of Hopf algebras carrying an induced Poisson structure which makes it into a Poisson Hopf algebra. For instance, the graded algebra associated to any connected Hopf algebra is such an example [24]. It is therefore important to achieve a good understanding of these structures as well as to be able to construct new ones. The present paper is a contribution to the study of Poisson Hopf algebras from an algebraic point of view. More precisely, in light of the increasing interest shown recently in the category of Hopf algebras (see $[2,3,4,5,8,17,18,19,23]$ and the references therein), we will study the categorical properties of Poisson Hopf algebras. As we will see, the category $k$-PoissBiAlg of Poisson bialgebras (and therefore the category of Poisson Hopf algebras) is not as friendly as the category $k$-BiAlg of

2010 Mathematics Subject Classification. 18A30, 18A35, 18A40.

Key words and phrases. bialgebra, Hopf algebra, Poisson Hopf algebra, coproduct, colimit, cocomplete, (co)refective subcategory, generators.

The author is supported by an Aspirant Fellowship from the Fund for Scientific Research-Flanders (Belgium) (F.W.O. Vlaanderen). This research is part of the grant no.88/05.10.2011 of the Romanian National Authority for Scientific Research, CNCS-UEFISCDI. 
bialgebras in the sense that it does not enjoy the same nice symmetry. More precisely, it is well-known that for any field $k$ the category $k$-BiAlg of bialgebras is isomorphic to $\operatorname{Mon}\left(\operatorname{Comon}\left({ }_{k} \mathcal{M}\right)\right)$ as well as to $\operatorname{Comon}\left(\operatorname{Mon}\left({ }_{k} \mathcal{M}\right)\right)$ while the category $k$-PoissBiAlg is only isomorphic to $\operatorname{Comon}(k-$ Poiss $)$, where $\operatorname{Mon}(\mathcal{C})$ and $\operatorname{Comon}(\mathcal{C})$ denote the monoids, respectively the comonoids, of a given category $\mathcal{C}$ (see for instance [19]) and $k$-Poiss stands for the category of Poisson algebras. Therefore, the main drawback in studying Poisson Hopf algebras is that the duality arguments usually employed for Hopf algebras do not hold anymore.

An outline of the paper is as follows. In Section 1 we introduce the notation and recall briefly the basic concepts needed in the sequel. Section 2 contains the explicit description of coproducts and coequalizers in the categories of Poisson algebras, Poisson bialgebras as well as Poisson Hopf algebras. As we will see, all these constructions rely on those performed in the category of Poisson algebras. The main results of the paper, namely the explicit constructions of the free Poisson Hopf algebra generated by a coalgebra, respectively a Poisson bialgebra, are proved in Section 3. The constructions in this section parallel those in [21]. However, it is worth pointing out that the construction of the free Poisson Hopf algebra on a coalgebra does not follow trivially from Takeuchi's construction. What we mean, precisely, is that we do not merely put a Lie algebra structure on Takeuchi's free Hopf algebra, the free Poisson Hopf algebra on a Poisson bialgebra being obtained by a different construction. The paper ends with some open problems concerning the existence of limits and cofree objects in the above mentioned categories. Another important issue, also connected to the existence of cofree objects, which still needs to be addressed is the injectivity (resp. surjectivity) of monomorphisms (resp. epimorphisms) in the category of Poisson Hopf algebras.

\section{Preliminaries}

Throughout this paper, $k$ will be a field. Unless specified otherwise, all vector spaces, tensor products, homomorphisms, algebras, coalgebras, bialgebras, Lie algebras, Poisson algebras, Hopf algebras and Poisson Hopf algebras are over $k$ and all algebras are considered to be unital. Our notation for the standard categories is as follows: ${ }_{k} \mathcal{M}(k-$ vector spaces), $k$-Alg (associative unital $k$-algebras), $k$-Lie (Lie algebras over $k$ ), $k$-Poiss (Poisson algebras over $k$ ), $k$-BiAlg (bialgebras over $k$ ), $k$-HopfAlg (Hopf algebras over $k$ ), $k$-BiAlgPoiss (Poisson bialgebras over $k$ ), $k$-HopfPoiss (Poisson Hopf algebras over $k$ ). For a coalgebra $C$, we will use Sweedler's $\Sigma$-notation $\Delta(c)=c_{(1)} \otimes c_{(2)}$ with suppressed summation sign. $C^{\text {cop }}$ stands for the coopposite of the coalgebra $C$. For a coalgebra $C$ and an algebra $A, \operatorname{Hom}_{k}(C, A)$ becomes an algebra with respect to the convolution product $*$; more precisely, we have $(f * g)(c)=f\left(c_{(1)}\right) g\left(c_{(2)}\right)$. If $A$ is an algebra then the vector space $A$ together with the product $[-,-]: A \times A \rightarrow A$ defined by $[a, b]=a b-b a$ is a Lie algebra denoted by $A^{-}$. Given a vector space $V,(T(V), i)$ stands for the tensor algebra on $V$, where $i: V \rightarrow T(V)$ is the canonical inclusion. The Lie subalgebra of $T(V)^{-}$generated by $i(V)$ is called the free Lie algebra generated by $V$ and will be denoted by $L(V)$. The symmetric algebra on $V$ will be denoted by $(S(V), i)$. If $\mathfrak{g}$ is a Lie algebra with bracket $[\cdot, \cdot]$ then the symmetric algebra $S(\mathfrak{g})$ inherits a Poisson algebra 
structure $\{\cdot, \cdot\}$ in a canonical way, namely $\{g, h\}=[g, h]$ for all $g, h \in \mathfrak{g}$. Furthermore, we denote by $(\mathcal{P}(V), \bar{i})$ the free Poisson algebra on $V$, where $\mathcal{P}(V)=S(L(V))$ and $\bar{i}: V \rightarrow \mathcal{P}(V)$ is the canonical inclusion; see [13] and the references therein for more details on the free Poisson algebra. As the terminology suggests, the functor sending a vector space $V$ to the free Poisson algebra $\mathcal{P}(V)$ provides a left adjoint to the forgetful functor $U: k$-Poiss $\rightarrow{ }_{k} \mathcal{M}$.

Let us recall briefly some well known results pertaining to category theory, referring the reader to $[1,12]$ for more details. A category $\mathcal{C}$ is called (co)complete if all diagrams in $\mathcal{C}$ have (co)limits in $\mathcal{C}$. A category $\mathcal{C}$ is (co) complete if and only if $\mathcal{C}$ has (co)equalizers of all pairs of arrows and all (co)products [15, Theorem 6.10]. For a more detailed discussion concerning the completeness and cocompleteness of some of the categories mentioned above we refer the reader to $[2,3,17,18]$. A full subcategory $\mathcal{D}$ of $\mathcal{C}$ is called (co)reflective in $\mathcal{C}$ when the inclusion functor $U: \mathcal{D} \rightarrow \mathcal{C}$ has a (right) left adjoint. A very convenient way of proving, in a constructive way, that a given covariant functor $F: \mathcal{C} \rightarrow \mathcal{D}$ has a left adjoint is by showing that for any object $X \in \mathcal{D}$ the co-universal problem generated by $X$ and $F$ has a co-universal solution. More precisely, given $X \in \mathcal{D}$, a co-universal solution for the co-universal problem generated by $X$ and $F$ consists of an object $G(X) \in \mathcal{C}$ and a map $i: X \rightarrow F(G(X))$ in $\mathcal{D}$ such that for each $Y \in \mathcal{C}$ and for each map $f: X \rightarrow F(Y)$ in $\mathcal{D}$ there is a unique map $g: G(X) \rightarrow Y$ in $\mathcal{C}$ such that the following diagram commutes:

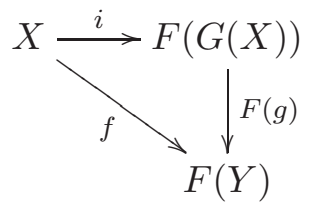

If for each $X \in \mathcal{D}$ the co-universal problem defined by $X$ and $F$ has a co-universal solution, then $G: \mathcal{D} \rightarrow \mathcal{C}$ defines a functor which is a left adjoint to $F$ [14].

Recall that a Poisson algebra is both an associative commutative algebra and a Lie algebra living on the same vector space $P$ such that any hamiltonian $[p,-]: P \rightarrow P$ is a derivation of the associative algebra $\mathrm{P}$, i.e. for all $p, q, r \in P$ we have:

$$
[p, q r]=[p, q] r+q[p, r]
$$

If $P_{1}, P_{2}$ are Poisson algebras then $P_{1} \otimes P_{2}$ has a Poisson algebra structure defined for all $p, r \in P_{1}$ and $q, s \in P_{2}$ by:

(1) $(p \otimes q) \cdot(r \otimes s):=p r \otimes q s, \quad[p \otimes q, r \otimes s]_{P \otimes P}:=p r \otimes[q, s]+[p, r] \otimes q s$

A linear map $f: P_{1} \rightarrow P_{2}$ is called a morphism of Poisson algebras if $f$ is both an algebra map as well as a Lie algebra map. Furthermore, the category $k$-Poiss of Poisson algebras is in fact a monoidal category with the tensor product defined above. A Poisson ideal is a linear subspace which is both an ideal with respect to the associative product as well as a Lie ideal. If $\mathcal{I}$ is a Poisson ideal of $P$ then $P / \mathcal{I}$ inherits a Poisson algebra structure in the obvious way.

A commutative bialgebra $B$ together with a Poisson bracket $[\cdot, \cdot]_{B}$ is called a Poisson bialgebra if the comultiplication $\Delta_{B}$ and the counit $\varepsilon_{B}$ are Poisson algebra maps, i.e. 
besides from being algebra maps, for all $a, b \in B$ we also have:

$$
\Delta_{B}([a, b])=\left[\Delta_{B}(a), \Delta_{B}(b)\right]_{B \otimes B}, \quad \varepsilon_{B}([a, b])=\left[\varepsilon_{B}(a), \varepsilon_{B}(b)\right]_{k}
$$

Let us observe that the second compatibility in (2) is trivially fulfilled as for all $a, b \in B$ we have $\varepsilon_{B}([a, b])=0$ (see [11]). Furthermore, if $B$ is a Hopf algebra then $B$ is called a Poisson Hopf algebra. It is straightforward to see that the antipode $S_{B}$ is a Poisson algebra anti-morphism, i.e. for all $a, b \in B$ we have: $S_{B}\left([a, b]_{B}\right)=\left[S_{B}(b), S_{B}(a)\right]_{B}$. A morphism of Poisson bialgebras is both a morphism of Poisson algebras and a morphism of coalgebras. A morphism of Poisson bialgebras between two Poisson Hopf algebras is automatically a Poisson Hopf morphism and therefore $k$-HopfPoiss is a full subcategory of the category $k$-BiAlgPoiss. It is straightforward to see that if $(H, m, \eta, \Delta, \varepsilon, S,[-,-])$ is a Poisson Hopf algebra then $\left(H^{\mathrm{cop}}, m, \eta, \Delta, \varepsilon, S,[-,-]^{\mathrm{op}}\right)$, where $[a, b]^{\mathrm{op}}=[b, a]$, is again a Poisson Hopf algebra which we will denote by $H^{\text {op,cop }}$. We refer to [20] for further details concerning Hopf algebras and to [10] for a comprehensive treatment of Poisson algebras from both algebraic and geometrical point of view.

\section{Colimits in the Category of Poisson Hopf algebras}

The aim of this section is to construct coproducts and coequalizers in the category $k$ PoissHopf of Poisson Hopf algebras. Since there is a close connection between these constructions and the ones corresponding to the category $k$-Poiss of Poisson algebras we start by investigating the latter category. Our next result constructs coproducts and coequalizers in the category of Poisson algebras; as we will see, these constructions are obtained by modifying properly the objects which provide the coproducts respectively the coequalizers in the category $k$-Alg of algebras [14, page 50].

Proposition 2.1. The category k-Poiss of Poisson algebras has arbitrary coproducts and coequalizers. In particular, the category $k$-Poiss of Poisson algebras is cocomplete.

Proof. We first indicate the construction of coproducts. Let $\left(P_{l}\right)_{l \in I}$ be a family of Poisson algebras and consider $\left(\bigoplus_{l \in I} P_{l},\left(j_{l}\right)_{l \in I}\right)$ to be their coproduct in ${ }_{k} \mathcal{M}$. Then $\left(\coprod_{l \in I} P_{l}:=\right.$ $\left.S\left(\bigoplus_{l \in I} P_{l}\right) / \bar{J},\{-,-\},\left(q_{l}\right)_{l \in I}\right)$ is the coproduct of the above family in $k$-Poiss, where $\left(S\left(\bigoplus_{l \in I} P_{l}\right), i\right)$ is the symmetric algebra on the vector space $\bigoplus_{l \in I} P_{l}, i: \bigoplus_{l \in I} P_{l} \rightarrow$ $S\left(\bigoplus_{l \in I} P_{l}\right)$ stands for the canonical inclusion, $\bar{J}$ is the Poisson ideal generated by the set $J:=\left\{i \circ j_{l}\left(x_{l} y_{l}\right)-i\left(j_{l}\left(x_{l}\right)\right) i\left(j_{l}\left(y_{l}\right)\right), 1_{S}\left(\oplus P_{l}\right)-i \circ j_{l}\left(1_{P_{l}}\right) \mid x_{l}, y_{l} \in P_{l}, l \in I\right\}, \nu$ : $S\left(\bigoplus_{l \in I} P_{l}\right) \rightarrow S\left(\bigoplus_{l \in I} P_{l}\right) / \bar{J}$ denotes the canonical projection and $q_{l}=\nu \circ i \circ j_{l}$, for all $l \in I$. Since the idea behind this construction is essentially the same as the one used in the case of associative algebras we will be brief. Consider $Q$ to be a Poisson algebra and $u_{r}: P_{r} \rightarrow Q$ a family of Poisson algebra maps. Composing the universal maps depicted in (3) it yields a unique Poisson algebra map $u: S\left(\bigoplus_{l \in I} P_{l}\right) / \bar{J} \rightarrow Q$ such that

$u \circ q_{r}=u_{r}$ : 


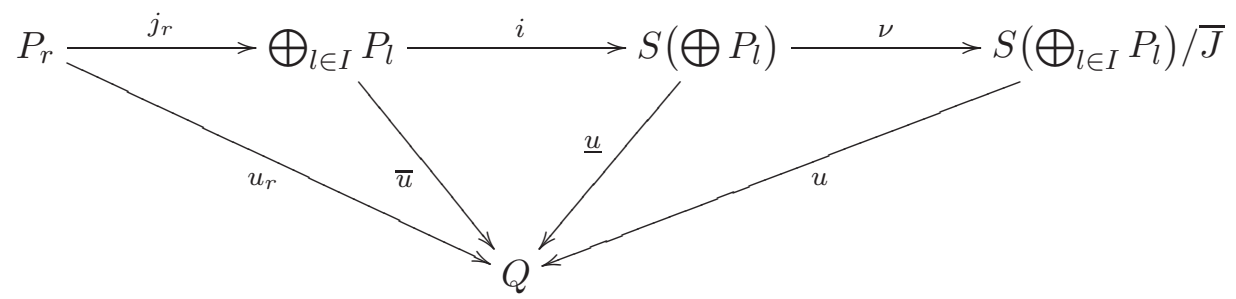

Next in line are coequalizers. Let $f, g: P \rightarrow Q$ be two Poisson algebra maps and consider $\bar{I}$ to be the Poisson ideal generated by the set $\{f(p)-g(p) \mid p \in P\}$. Then $(Q / \bar{I}, \pi)$ is the coequalizer of the morphisms $(f, g)$ in $k$-Poiss, where $\pi: Q \rightarrow Q / \bar{I}$ is the canonical projection; the details are straightforward and left to the reader.

In order to have a complete picture on the category $k$-Poiss of Poisson algebras we record here the result concerning limits:

Proposition 2.2. The category k-Poiss of Poisson algebras has arbitrary products and equalizers. In particular, the category $k$-Poiss of Poisson algebras is complete.

Proof. It is straightforward to see that both products as well as equalizers can be constructed as simply the products and respectively the equalizers of the underlying vector spaces with the obvious commutative algebra and Lie algebra structures.

As mentioned before, the colimits construction in the categories $k$-BiAlgPoiss and $k$ HopfPoiss rely heavily on the corresponding construction performed in the category $k$-Poiss.

Theorem 2.3. The categories $k$-BiAlgPoiss and $k$-HopfPoiss of Poisson bialgebras and respectively Poisson Hopf algebras have arbitrary coproducts and coequalizers. In particular, the above categories are cocomplete.

Proof. First we deal with products in the category of Poisson bialgebras. Consider a family of Poisson bialgebras $\left(B_{i}, m_{i}, \eta_{i}, \Delta_{i}, \varepsilon_{i},[-,-]_{i}\right)_{i \in I}$ and let $\left(\coprod_{i \in I} B_{i},\left(q_{i}\right)_{i \in I}\right)$ be the coproduct of this family in the category $k$-Poiss as described in Proposition 2.1. It turns out that $\coprod_{i \in I} B_{i}$ is actually a Poisson bialgebra with comultiplication and counit given by the unique Poisson algebra maps such that the following diagrams commute:
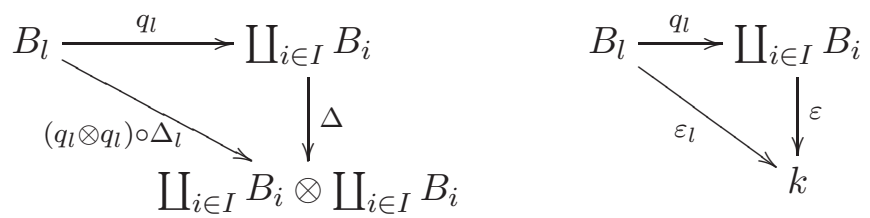

Proving that $\left(\coprod_{i \in I} B_{i}, \Delta, \varepsilon\right)$ is a coalgebra goes essentially in the same vain as in the case of Hopf algebras; we refer the reader to [14] for more details.

Next we look at coequalizers. Let $f, g: P \rightarrow Q$ be two Poisson bialgebra maps and consider $\bar{I}$ to be the Poisson ideal of $Q$ generated by the set $\{f(p)-g(p) \mid p \in P\}$. We prove that $\bar{I}$ is also a coideal, i.e. $\Delta(\bar{I}) \subseteq Q \otimes \bar{I}+\bar{I} \otimes Q$. In fact, as $\Delta$ is a Poisson algebra 
morphism we only need to check that $\Delta([q, r(f(p)-g(p))]) \subseteq Q \otimes \bar{I}+\bar{I} \otimes Q$, for all $q, r \in Q$ and $p \in P$. Indeed, using induction this would imply that $\Delta\left(\left[q_{1},\left[q_{2}, \ldots,\left[q_{k}, r(f(p)-\right.\right.\right.\right.$ $g(p))]]]) \subseteq Q \otimes \bar{I}+\bar{I} \otimes Q$ for all $k \in \mathbb{N}, q_{1}, q_{2}, \ldots, q_{k}, r \in Q, p \in P$ and the conclusion follows. Now we prove our original claim:

$$
\begin{aligned}
& \Delta([q, r(f(p)-g(p))])=[\Delta(q), \Delta(r(f(p)-g(p)))]_{Q \otimes Q} \\
& =\left[q_{(1)} \otimes q_{(2)},\left(r_{(1)} \otimes r_{(2)}\right)\left(f\left(p_{(1)}\right) \otimes f\left(p_{(2)}\right)-g\left(p_{(1)}\right) \otimes g\left(p_{(2)}\right)\right)\right]_{Q \otimes Q} \\
& =\left[q_{(1)} \otimes q_{(2)},\left(r_{(1)} \otimes r_{(2)}\right)\left(f\left(p_{(1)}\right) \otimes\left(f\left(p_{(2)}\right)-g\left(p_{(2)}\right)\right)\right)-\right. \\
& \left.\left(r_{(1)} \otimes r_{(2)}\right)\left(\left(f\left(p_{(1)}\right)-g\left(p_{(1)}\right)\right) \otimes g\left(p_{(2)}\right)\right)\right]_{Q \otimes Q} \\
& =\left[q_{(1)} \otimes q_{(2)}, r_{(1)} f\left(p_{(1)}\right) \otimes r_{(2)}\left(f\left(p_{(2)}\right)-g\left(p_{(2)}\right)\right)\right]_{Q \otimes Q}- \\
& {\left[q_{(1)} \otimes q_{(2)}, r_{(1)}\left(f\left(p_{(1)}\right)-g\left(p_{(1)}\right)\right) \otimes r_{(2)} g\left(p_{(2)}\right)\right]_{Q \otimes Q}} \\
& \stackrel{(1)}{=} q_{(1)} r_{(1)} f\left(p_{(1)}\right) \otimes\left[q_{(2)}, r_{(2)}\left(f\left(p_{(2)}\right)-g\left(p_{(2)}\right)\right)\right]+ \\
& {\left[q_{(1)}, r_{(1)} f\left(p_{(1)}\right)\right] \otimes q_{(2)} r_{(2)}\left(f\left(p_{(2)}\right)-g\left(p_{(2)}\right)\right)-} \\
& q_{(1)} r_{(1)}\left(f\left(p_{(1)}\right)-g\left(p_{(1)}\right)\right) \otimes\left[q_{(2)}, r_{(2)} g\left(p_{(2)}\right)\right]- \\
& {\left[q_{(1)}, r_{(1)}\left(f\left(p_{(1)}\right)-g\left(p_{(1)}\right)\right)\right] \otimes q_{(2)} r_{(2)} g\left(p_{(2)}\right)}
\end{aligned}
$$

and the last line is obviously in $Q \otimes \bar{I}+\bar{I} \otimes Q$, as the underlined terms are in $\bar{I}$. Therefore, $Q / \bar{I}$ is a Poisson bialgebra in a canonical way and moreover $(Q / \bar{I}, \pi)$ is the coequalizer of the morphisms $(f, g)$ in $k$-BiAlgPoiss, where $\pi: Q \rightarrow Q / \bar{I}$ is the canonical projection. Consider now a family of Poisson Hopf algebras $\left(H_{i}, m_{i}, \eta_{i}, \Delta_{i}, \varepsilon_{i}, S_{i},[-,-]_{i}\right)_{i \in I}$ and let $\left(H:=\coprod_{i \in I} H_{i}, m, \eta, \Delta, \varepsilon,[-,-],\left(q_{i}\right)_{i \in I}\right)$ be the previously constructed coproduct of the underlying Poisson bialgebras. Remark that $S_{i}: H_{i} \rightarrow H_{i}{ }^{\text {op,cop }}$ is a Poisson bialgebra map. Then, the universal property of the coproduct yields an unique Poisson bialgebra map $S: H \rightarrow H^{\text {op,cop }}$ such that the following diagram commutes for all $i \in I$ :

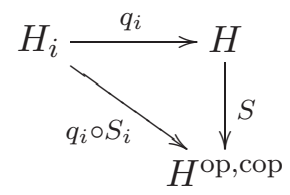

As $S: H \rightarrow H^{\text {op,cop }}$ defined in (5) is a Poisson bialgebra map we only need to prove that $S$ is indeed an antipode for $H$. This follows exactly as in the proof of [3, Theorem 2.2]. Finally, since $k$-HopfPoiss is a full subcategory of the category $k$-BiAlgPoiss it follows that $\left(H:=\coprod_{i \in I} H_{i}, \Delta, \varepsilon, S,[-,-],\left(q_{i}\right)_{i \in I}\right)$ is also the coproduct in $k$-HopfPoiss.

Consider now $f, g: P \rightarrow Q$ to be two Poisson Hopf algebra maps and, as before, let $\bar{J}$ be the Poisson ideal of $Q$ generated by the set $\{f(p)-g(p) \mid p \in P\}$. The computations performed in the case of Poisson bialgebras imply that $\bar{j}$ is also a coideal. We are left to prove that $\bar{j}$ is a Hopf ideal, i.e. $S_{Q}(\bar{J}) \subseteq \bar{J}$. Arguing as in the first part of the proof, we only need to show that $S_{Q}([q, r(f(p)-g(p))) \subseteq \bar{J}$ for all $q, r \in Q$ and $p \in P$. Indeed, 
we have:

$$
\begin{aligned}
S_{Q}([q, r(f(p)-g(p))) & =\left[S_{Q}(r(f(p)-g(p))), S_{Q}(q)\right] \\
& =\left[S_{Q}(f(p)-g(p)) S_{Q}(r), S_{Q}(q)\right] \\
& =\left[\left(f\left(S_{P}(p)\right)-g\left(S_{P}(p)\right)\right) S_{Q}(r), S_{Q}(q)\right] \\
& =-\left[S_{Q}(q), S_{Q}(r)\left(f\left(S_{P}(p)\right)-g\left(S_{P}(p)\right)\right)\right] \in \bar{J}
\end{aligned}
$$

and the proof is now finished.

\section{The free Poisson Hopf algebra on a coalgebra}

In this section we introduce the main characters of this paper, namely the free Poisson Hopf algebras generated by coalgebras. The strategy we pursue is the following: first, we introduce the free Poisson bialgebra on a coalgebra (Theorem 3.1) and then we prove that there also exist a free Poisson Hopf algebra on every Poisson bialgebra (Theorem 3.2). Finally, by putting the two constructions together we arrive at the free Poisson Hopf algebra generated by a coalgebra (Theorem 3.3).

Theorem 3.1. The forgetful functor $F_{1}: k$-BiAlgPoiss $\rightarrow k$-CoAlg has a left adjoint, i.e. there exists a free Poisson bialgebra on every coalgebra.

Proof. Let $\left(C, \Delta_{C}, \varepsilon_{C}\right)$ be a coalgebra and consider $(\mathcal{P}(C), \bar{i})$ to be the free Poisson algebra on the vector space $C$. By the universal property of the free Poisson algebra we obtain two Poisson algebra maps $\bar{\Delta}: \mathcal{P}(C) \rightarrow \mathcal{P}(C) \otimes \mathcal{P}(C)$ and respectively $\bar{\varepsilon}: \mathcal{P}(C) \rightarrow$ $k$ such that the following two diagrams commute:

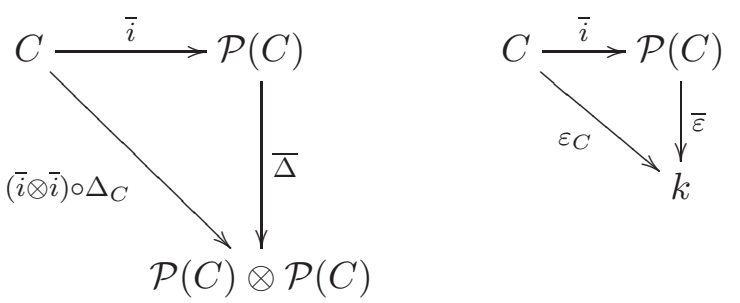

We start by proving that $(\mathcal{P}(C), \bar{\Delta}, \bar{\varepsilon})$ is a coalgebra. Consider the Poisson algebra map $(\bar{\Delta} \otimes I d) \circ \bar{\Delta} \circ \bar{i}$. The universal property of the free Poisson algebra yields a unique Poisson algebra map $\psi: \mathcal{P}(C) \rightarrow \mathcal{P}(C) \otimes \mathcal{P}(C) \otimes \mathcal{P}(C)$ such that the following diagram commutes:

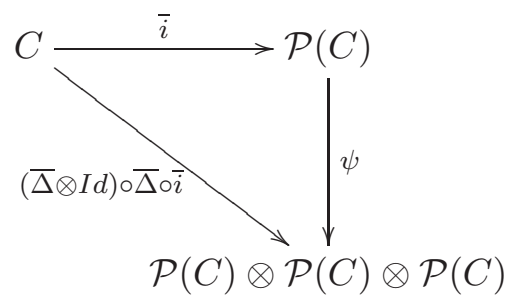

It is easy to see that the Poisson algebra map $(\bar{\Delta} \otimes I d) \circ \bar{\Delta}$ makes the above diagram commute. Thus, using the uniqueness of $\psi$, in order to prove that $(\bar{\Delta} \otimes I d) \circ \bar{\Delta}=$ 
$(I d \otimes \bar{\Delta}) \circ \bar{\Delta}$ it is enough to show that $(\bar{\Delta} \otimes I d) \circ \bar{\Delta} \circ \bar{i}=(\operatorname{Id} \otimes \bar{\Delta}) \circ \bar{\Delta} \circ \bar{i}$. Indeed, concerning the last claim we have:

$$
\begin{aligned}
(\bar{\Delta} \otimes I d) \circ \bar{\Delta} \circ \bar{i} & \stackrel{(6)}{=}(\bar{\Delta} \otimes I d) \circ(\bar{i} \otimes \bar{i}) \circ \Delta_{C} \\
= & ((\underline{\underline{\Delta} \circ \bar{i}}) \otimes \bar{i}) \circ \Delta_{C} \\
& \stackrel{(6)}{=}\left(\left((\bar{i} \otimes \bar{i}) \circ \Delta_{C}\right) \otimes \bar{i}\right) \circ \Delta_{C} \\
= & (\bar{i} \otimes \bar{i} \otimes \bar{i}) \circ \underline{\left(I d \otimes \Delta_{C}\right) \circ \Delta_{C}} \\
= & (\bar{i} \otimes \bar{i} \otimes \bar{i}) \circ\left(\Delta_{C} \otimes I d\right) \circ \Delta_{C} \\
= & \left(\left(\bar{i} \otimes \underline{\left.(\bar{i} \otimes \bar{i}) \circ \Delta_{C}\right)}\right) \circ \Delta_{C}\right. \\
& \stackrel{(6)}{=}(\bar{i} \otimes(\bar{\Delta} \circ \bar{i})) \circ \Delta_{C} \\
= & (I d \otimes \bar{\Delta}) \circ \underline{(\bar{i} \otimes \bar{i}) \circ \Delta_{C}} \\
& \stackrel{(6)}{=} \quad(I d \otimes \bar{\Delta}) \circ \bar{\Delta} \circ \bar{i}
\end{aligned}
$$

A similar argument proves that $(I d \otimes \bar{\varepsilon}) \circ \bar{\Delta}=(\bar{\varepsilon} \otimes I d) \circ \bar{\Delta}=I d$ and thus $\mathcal{P}(C)$ is in fact a Poisson bialgebra. The proof will be finished once we show that the pair $(\mathcal{P}(C), \bar{i})$ provides a co-universal solution to the co-universal problem generated by the coalgebra $C$ and the forgetful functor $F_{1}: k$-BiAlgPoiss $\rightarrow k$-CoAlg. To this end, let $H$ be a Poisson bialgebra and $f: C \rightarrow H$ a coalgebra map. By the universal property of the free Poisson algebra, there exists a Poisson algebra map $\bar{f}: \mathcal{P}(C) \rightarrow H$ such that the following diagram is commutative:

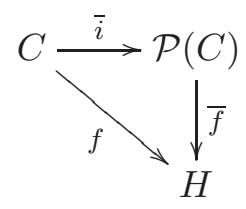

We are left to prove that $\bar{f}$ is also a coalgebra map. Since $\Delta_{H} \circ \bar{f}$ is a Poisson algebra map, by the universal property of the free Poisson algebra, there exists a unique Poisson algebra map $\xi: \mathcal{P}(C) \rightarrow H \otimes H$ such that the following diagram commutates:

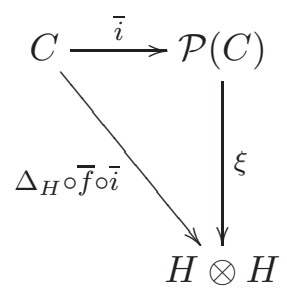

Obviously, $\Delta_{H} \circ \bar{f}$ makes the above diagram commutative. By the same argument as before, in order to prove that $(\bar{f} \otimes \bar{f}) \circ \bar{\Delta}=\Delta_{H} \circ \bar{f}$, it will be enough to show that 
$(\bar{f} \otimes \bar{f}) \circ \bar{\Delta} \circ \bar{i}=\Delta_{H} \circ \bar{f} \circ \bar{i}$. As for the last claim, we have:

$$
\begin{array}{rlrl}
(\bar{f} \otimes \bar{f}) \circ \bar{\Delta} \circ \bar{i} & \stackrel{(6)}{=} & (\bar{f} \otimes \bar{f}) \circ(\bar{i} \otimes \bar{i}) \circ \Delta_{C} \\
= & & (\overline{(\bar{f} \circ \bar{i}}) \otimes(\bar{f} \circ \bar{i})) \circ \Delta_{C} \\
& = & & (\overline{f \otimes f}) \circ \Delta_{C} \\
f \text { coalgebra map } & & \Delta_{H} \circ f=\Delta_{H} \circ \bar{f} \circ \bar{i}
\end{array}
$$

Consider now the Poisson algebra map $\varepsilon_{H} \circ \bar{f} \circ \bar{i}$. Again by the universal property of the free Poisson algebra, there exists a unique Poisson algebra map $\chi: \mathcal{P}(C) \rightarrow k$ such that the following diagram is commutative:

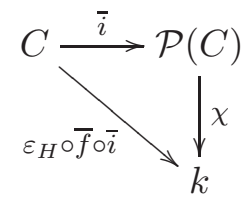

Using the same argument as before, in order to prove that $\varepsilon_{H} \circ \bar{f}=\bar{\varepsilon}$ we only need to show that $\varepsilon_{H} \circ \bar{f} \circ \bar{i}=\bar{\varepsilon} \circ \bar{i}$. The latter statement holds true by the following straightforward computation:

$$
\varepsilon_{H} \circ \bar{f} \circ \bar{i}=\varepsilon_{H} \circ f=\varepsilon_{C}=\bar{\varepsilon} \circ \bar{i}
$$

This finishes the proof.

In the proof of our next theorem we make use of some statements already proven in [14, Theorem 2.6.3]. Since the proof there is very detailed and freely available, we will refer to it and leave out those computations.

Theorem 3.2. The forgetful functor $F_{2}: k$-PoissHopf $\rightarrow k$-BiAlgPoiss has a left adjoint, i.e. there exists a free Poisson Hopf algebra on every Poisson bialgebra.

Proof. Let $B$ be a Poisson bialgebra. We aim to construct a co-universal solution to the co-universal problem generated by $B$ and the forgetful functor $F_{2}: k$-PoissHopf $\rightarrow k$-BiAlgPoiss. To this end, consider $\left(C=\coprod_{n \in \mathbb{N}} B_{i},\left(q_{n}\right)_{n \in \mathbb{N}}\right)$ to be the coproduct in $k$-BiAlgPoiss of the Poisson bialgebras $B_{n}, n \geq 0$, where $B_{n}=B$ for $n$ even and $B_{n}=B^{\text {op,cop }}$ for $n$ odd. The universality of the coproduct yields a unique Poisson bialgebra map $S^{\prime}: C \rightarrow C^{\text {op,cop }}$ which makes the following diagram commutative:

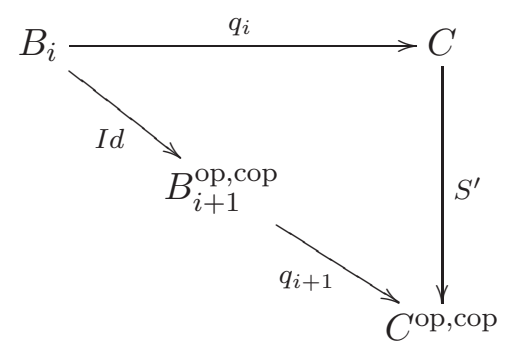

Now consider $\mathcal{I}$ to be the Poisson ideal of $C$ generated by the set $I=\left\{\left(S^{\prime} * I d-\right.\right.$ $\left.\left.u_{C} \circ \varepsilon_{C}\right)\left(q_{n}(x)\right),\left(I d * S^{\prime}-u_{C} \circ \varepsilon_{C}\right)\left(q_{n}(x)\right) \mid x \in B_{n}, n \in \mathbb{N}\right\}$. We will prove that $\mathcal{I}$ 
is also a Hopf ideal, i.e. $\varepsilon_{C}(\mathcal{I})=0, \Delta_{C}(\mathcal{I}) \subseteq C \otimes \mathcal{I}+\mathcal{I} \otimes C$ and $S^{\prime}(\mathcal{I}) \subseteq \mathcal{I}$. As noted in the proof of Theorem 2.3, it is enough to check this on the elements of the form $\left[a, b\left(S^{\prime}\left(q_{n}(x)_{(1)}\right) q_{n}(x)_{(2)}-u_{C} \circ \varepsilon_{C}\left(q_{n}(x)\right)\right)\right]$ where $a, b \in C$ and $x \in B_{n}, n \in \mathbb{N}$. We know from [14, Theorem 2.6.3] that $\Delta_{C}\left(S^{\prime}\left(q_{n}(x)_{(1)}\right) q_{n}(x)_{(2)}-u_{C}\right.$ ॰ $\left.\varepsilon_{C}\left(q_{n}(x)\right)\right) \in C \otimes C I+C I \otimes C$, where $C I$ denotes the two-sided ideal generated by $I$. Since the inclusion $C I \subset \mathcal{I}$ holds true, we will denote $\Delta_{C}\left(S^{\prime}\left(q_{n}(x)_{(1)}\right) q_{n}(x)_{(2)}-u_{C} \circ \varepsilon_{C}\left(q_{n}(x)\right)\right)=c \otimes \iota+\bar{\imath} \otimes \bar{c}$ (note that in order to be consistent with our notations, we suppressed the summation sign in the right hand side) with $c, \bar{c} \in C$ and $\iota, \bar{\imath} \in \mathcal{I}$. Then, we have:

$$
\begin{array}{ll} 
& \Delta_{C}\left(\left[a, b\left(S^{\prime}\left(q_{n}(x)_{(1)}\right) q_{n}(x)_{(2)}-u \circ \varepsilon\left(q_{n}(x)\right)\right)\right]_{C}\right)= \\
= & {\left[\Delta(a), \Delta(b) \Delta\left(S^{\prime}\left(q_{n}(x)_{(1)}\right) q_{n}(x)_{(2)}-u \circ \varepsilon\left(q_{n}(x)\right)\right)\right]_{C \otimes C}} \\
= & {\left[a_{(1)} \otimes a_{(2)},\left(b_{(1)} \otimes b_{(2)}\right)(c \otimes \iota+\bar{\imath} \otimes \bar{c})\right]_{C \otimes C}} \\
\stackrel{(1)}{=} & {\left[a_{(1)} \otimes a_{(2)}, b_{(1)} c \otimes b_{(2)} \iota\right]_{C \otimes C}+\left[a_{(1)} \otimes a_{(2)}, b_{(1)} \bar{\imath} \otimes b_{(2)} \bar{c}\right]_{C \otimes C}} \\
\stackrel{(1)}{=} & a_{(1)} b_{(1)} c \otimes \underline{\left[a_{(2)}, b_{(2)} \iota\right]_{C}}+\left[a_{(1)}, b_{(1)} c\right]_{C} \otimes \underline{a_{(2)} b_{(2)} \iota}+\underline{a_{(1)} b_{(1)} \bar{\imath} \otimes\left[a_{(2)}, b_{(2)} \bar{c}\right]_{C}} \\
& +\underline{\left[a_{(1)}, b_{(1)}\right]_{C} \otimes a_{(2)} b_{(2)}} \bar{c}
\end{array}
$$

and the conclusion follows since the underlined terms belong to $\mathcal{I}$. We are left to show that $S^{\prime}(\mathcal{I}) \subseteq \mathcal{I}$. Recall from the proof of [14, Theorem 2.6.3] that $S^{\prime}\left(S^{\prime}\left(q_{n}(x)_{(1)}\right) q_{n}(x)_{(2)}-\right.$ $\left.u_{C} \circ \varepsilon_{C}\left(q_{n}(x)\right)\right)=q_{n+1}(x)_{(1)} S^{\prime}\left(q_{n+1}(x)_{(2)}\right)-u_{C} \circ \varepsilon_{C}\left(q_{n+1}(x)\right) \in I$ for all $x \in B_{n}, n \in \mathbb{N}$. Therefore, we have:

$$
\begin{aligned}
& S^{\prime}\left(\left[a, b\left(S^{\prime}\left(q_{n}(x)_{(1)}\right) q_{n}(x)_{(2)}-u \circ \varepsilon\left(q_{n}(x)\right)\right)\right]_{C}\right)= \\
= & {\left[S^{\prime}\left(S^{\prime}\left(q_{n}(x)_{(1)}\right) q_{n}(x)_{(2)}-u \circ \varepsilon\left(q_{n}(x)\right)\right) S^{\prime}(b), S^{\prime}(a)\right]_{C} } \\
= & {\left[\underline{\left(q_{n+1}(x)_{(1)} S^{\prime}\left(q_{n+1}(x)_{(2)}\right)-u \circ \varepsilon\left(q_{n+1}(x)\right)\right)} S^{\prime} q_{n+1}(x)_{(1)} S^{\prime}(b), S^{\prime}(a)\right]_{C} }
\end{aligned}
$$

and the last line is clearly in $\mathcal{I}$ since the underlined term belongs to $I$.

We are now ready to construct a co-universal solution to the co-universal problem generated by the Poisson bialgebra $B$ and the functor $F_{2}: k$-PoissHopf $\rightarrow k$-BiAlgPoiss. To this end, consider the Poisson algebra $H(C):=C / \mathcal{I}$ and $\pi: C \rightarrow C / \mathcal{I}$ the canonical projection. $H(C)$ can be made into a Poisson Hopf algebra with the coalgebra structure $(\bar{\Delta}, \bar{\varepsilon})$ and antipode $\bar{S}$ given by the unique Poisson algebra maps which make the following diagrams commute:
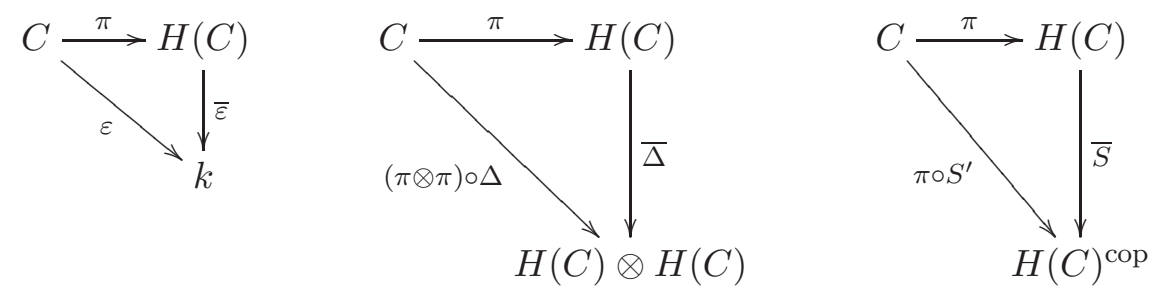
By arguments similar to those used in the proof of [14, Theorem 2.6.3] it can be easily seen that the object constructed above indeed provides a co-universal solution to the couniversal problem generated by the Poisson bialgebra $B$ and the functor $F_{2}: k$-PoissHopf $\rightarrow k$-BiAlgPoiss.

We can now put the last two results together:

Theorem 3.3. The forgetful functor $F: k$-PoissHopf $\rightarrow k$-CoAlg has a left adjoint, i.e. there exists a free Poisson Hopf algebra on every coalgebra.

Proof. It follows by composing the two left adjoint functors constructed in Theorem 3.1 and Theorem 3.2.

Corollary 3.4. The categories $k$-PoissHopf and k-BiAlgPoiss of Poisson Hopf algebras and respectively Poisson bialgebras have generators.

Proof. We denote by $\bar{F}_{1}: \mathrm{k}$-CoAlg $\rightarrow k$-BiAlgPoiss and $\bar{F}: \mathrm{k}$-CoAlg $\rightarrow k$-PoissHopf the left adjoint functors of $F_{1}: \mathrm{k}$-BiAlgPoiss $\rightarrow k$-CoAlg and respectively $F: k$-PoissHopf $\rightarrow k$-CoAlg. By [16, Theorem 17], the category $k$-CoAlg of coalgebras has a generator $G$. Recall that $G=\coprod_{x \in I} C_{x}$, where $I$ is the set of isomorphism classes of finite dimensional coalgebras over $k$ while $C_{x}$ is a coalgebra in the isomorphism class of $x \in I$. Now since both forgetful functors $F_{1}$ and $F$ are faithful it follows that $\bar{F}_{1}(G)$ and $\bar{F}(G)$ are generators in the categories of Poisson bialgebras and respectively Poisson Hopf algebras.

\section{SOME COMMENTS AND OPEN PROBLEMS}

The main results of this paper are Theorem 2.3 and Theorem 3.3 which prove the cocompleteness of the categories $k$-BiAlgPoiss and $k$-PoissHopf, and respectively the existence of a free Poisson Hopf algebra on every coalgebra. In order to complete the categorical picture drawn by the above results, it is natural to ask the following:

Question 1: Are the categories $k$-BiAlgPoiss and respectively $k$-PoissHopf complete (i.e. do they have arbitrary products and equalizers)?

It is worth pointing out that, if limits in $k$-BiAlgPoiss and respectively $k$-PoissHopf do exist, then Theorem 3.3 tells us that they should be constructed as simply the limits of the underlying coalgebras.

Question 2: Does there exist a cofree Poisson Hopf algebra on every Poisson algebra, respectively on every Poisson bialgebra? (i.e. do the forgetful functors $U: k$-PoissHopf $\rightarrow k$-Poiss and respectively $\bar{U}: k$-PoissHopf $\rightarrow k$-BiAlgPoiss have right adjoints?)

Another important issue, also related to the existence of cofree objects in the above mentioned categories, is the injectivity (resp. surjectivity) of monomorphisms (resp. epimorphisms). It was proven in [4] that monomorphisms (resp. epimorphisms) in the category $k$-HopfAlg of Hopf algebras are not necessarily injective (reps. surjective) maps. This was achieved by noticing that the antipode of any Hopf algebra is both a monomorphism and an epimorphism in the category $k$-HopfAlg of Hopf algebras together with 
the well known fact that there exist Hopf algebras with non-injective, respectively nonsurjective antipode. Although the result in [4] holds true in the category $k$-PoissHopf of Poisson Hopf algebras as well, it has no implications on the injectivity (resp. surjectivity) of monomorphisms (resp. epimorphisms) due to the fact that all Poisson Hopf algebras have bijective antipodes as a consequence of being commutative Hopf algebras.

As noted above, we do not know whether epimorphisms in the aforementioned categories are surjective maps. This information is of particular interest mainly in connection to the Special Adjoint Functor Theorem (see [12, Corollary p. 130]) which, under some additional assumptions, provides necessary and sufficient conditions for a functor to be a left (respectively right) adjoint. Having a positive answer to the question of whether epimorphisms in $k$-PoissHopf are surjective maps would also settle the co-wellpoweredness problem into positive and therefore all the assumptions required for applying the Special Adjoint Functor Theorem would be fulfilled. Thus, the forgetful functors $U$ and $\bar{U}$ would have right adjoints and therefore we would be able to construct the cofree Poisson Hopf algebra generated by a Poisson algebra, respectively a Poisson bialgebra. On the other hand, a negative answer to the question of whether epimorphisms are surjective maps in $k$-PoissHopf it has no implications on the co-wellpoweredness of the category $k$-PoissHopf: for instance, the category $k$-HopfAlg of Hopf algebras is co-wellpowered although epimorphisms are not necessarily surjective maps (see $[3,4,17]$ ).

\section{REFERENCES}

[1] Adámek, J., Rosický, J. - Locally Presentable and Accessible Categories, Cambridge University Press, 1994

[2] Agore, A.L. - Limits of coalgebras, bialgebras and Hopf algebras, Proc. Amer. Math. Soc., 139 (2011), 855-863

[3] Agore, A.L. - Categorical constructions for Hopf algebras, Comm. Algebra., 39 (2011), 1476-1481

[4] Chirvasitu, A. - On epimorphisms and monomorphisms of Hopf algebras, J. Algebra, 323 (2010), 1593-1606

[5] Chirvasitu, A. - Categorical aspects of compact quantum groups, Appl. Categ. Structures, DOI 10.1007/s10485-013-9333-8, arXiv:1208.5193.

[6] Drinfeld, V. - Quantum groups, Proc. Intern. Congr. Math., Berkeley, Vol. I (1987), 798 - 820

[7] Gavarini, F. - Poisson geometrical symmetries associated to non-commutative formal diffeomorphisms, Comm. Math. Phys. 253 (2005), 121-155

[8] Kawamura, K. - Inductive limit violates quasi-cocomutativity, Appl. Cat. Structures 21 (2013), $837-849$

[9] Korogodski, L.I. and Soibelman, Y.S. - Algebras of functions on quantum groups, Part I, Mathematical surveys and monographs 56, AMS 1998

[10] Laurent-Gengoux, C., Pichereau, A. and Vanhaecke, P. - Poisson structures, Grundlehren der mathematischen Wissenschaften 347 Springer, Heidelberg, 2013

[11] Lu, J., Wang, X. and Zhuang, G. - Universal enveloping algebras of Poisson Hopf algebras, preprint, arXiv:1402.2007

[12] Mac Lane, S. - Categories for the working mathematician, GTM 5, Springer, New York (1998)

[13] Makar-Limanov, L. and Shestakov, I. - Polynomial and Poisson dependence in free Poisson algebras and free Poisson fields, J. Algebra, 349 (2012), 372-379

[14] Pareigis, B. - Lectures on quantum groups and noncommutative geometry. Available at: http://www.mathematik.uni-muenchen.de/ pareigis/Vorlesungen/02SS/QGandNCG.pdf

[15] Pareigis, B. - Advanced Algebra. Available at:

http://www.mathematik.uni-muenchen.de/ pareigis/Vorlesungen/01WS/advalg.pdf 
[16] Pareigis, B., Sweedler, M.E. - On generators and cogenerators, Manuscripta Math., 2 (1970), 49-66

[17] Porst, H.-E. - Universal constructions for Hopf algebras, J. Pure Appl. Algebra, 212 (2008), 25472554

[18] Porst, H.-E. - Takeuchi's free Hopf algebra construction revisited, J. Pure Appl. Algebra, 216 (2012), $1768-1774$

[19] Porst, H.-E. - The Formal Theory of Hopf Algebras, Part II, preprint 2013.

[20] Sweedler, M.E. - Hopf Algebras, Benjamin New York, 1969

[21] Takeuchi, M. - Free Hopf algebras generated by coalgebras, J. Math. Soc. Japan 23 (1971), 561-582

[22] Tanisaki, T. - Poisson Hopf algebras associated to quantized enveloping algebras, preprint, arXiv:0802.1590v2

[23] Wisbauer, R. - Categorical aspects of Hopf algebras, in: Quantum groups and noncommutative spaces, Vieweg und Teubner (2011), 146-163

[24] Zhuang, G. - Properties of connected Hopf algebras of finite Gelfand-Kirillov dimension, J. London Math. Soc., 87(2013), 877-898

Faculty of Engineering, Vrije Universiteit Brussel, Pleinlaan 2, B-1050 Brussels, Belgium and Department of Applied Mathematics, Bucharest University of Economic Studies, Piata Romana 6, RO-010374 Bucharest 1, Romania

E-mail address: ana.agore@vub.ac.be and ana.agore@gmail.com 